\title{
The interstellar medium local to HD 10125 (O9.7 II)
}

\author{
Silvina Cichowolski ${ }^{1}$, E. Marcelo Arnal ${ }^{1,2}$, Cristina E. Cappa ${ }^{1,2}$, \\ Sergey Pineault ${ }^{3,4}$, and Nicole St-Louis ${ }^{4,5}$ \\ ${ }^{1}$ Instituto Argentino de Radioastronomía, \\ C.C. 5, 1894 Villa Elisa, Buenos Aires, Argentina \\ ${ }^{2}$ Facultad de Ciencias Astronómicas y Geofísicas, \\ Universidad Nacional La Plata, 1900 La Plata, Argentina \\ ${ }^{3}$ Département de Physique, Université Laval, \\ Ste-Foy, Québec G1K 7P4, Canada \\ ${ }^{4}$ Observatoire du Mont Mégantic, Notre-Dame-des-Bois, Canada \\ ${ }^{5}$ Département de Physique, Université de Montréal, \\ CP6128, Succursale Centre Ville, Montréal, Québec H3C 3J7, Canada
}

\section{Introduction}

The structure and dynamics of the interstellar medium (ISM) are strongly affected by the action of massive stars. They deposit in the ISM a huge number of ionizing photons and transfer to it vast amounts of mechanical energy via their powerful stellar winds. As a consequence, massive stars create what is known as an interstellar bubble, i.e., a minimum in the gas distribution characterized by a low volume density and a high temperature, surrounded by an expanding envelope (Weaver et al. 1977). The star should be seen projected onto, or close to, the centre of the $\mathrm{H}$ I minimum.

Observationally, one possible way to study this interaction is by analyzing the $\mathrm{H} \mathrm{I}$ and $\mathrm{HI}$ gas distribution in the environment of these stars. The radiation emitted by hot, massive stars can be absorbed by the interstellar dust which is heated, so an analysis of the infrared emission is also useful.

Using the Canadian Galactic Plane Survey (CGPS) database (Taylor 2001), IRAS-HIRES data and single-dish continuum surveys at $2695 \mathrm{MHz}$ and $4850 \mathrm{MHz}$, we analyzed the ISM local to the Galactic O-type star HD 10125. This star is classified as O9.5 Ib by Münch (1957) and as O9.7 II by Walborn (1971), and is located at $(l, b)=\left(128^{\circ} 29,+1^{\circ} .82\right)$. Its distance determinations range from 2.7 to $3.4 \mathrm{kpc}$ and its proper motion amounts to $\left(\mu_{\alpha}, \mu_{\delta}\right)=(4 \pm 1.6,1.8 \pm 1.6)$ mas yr$^{-1}$ (Tycho-2 Catalogue).

\section{Results}

From the $21 \mathrm{~cm}$ line data, an $\mathrm{H} \mathrm{I}$ minimum of about $18 \mathrm{pc}$ in diameter is found in the velocity range -27 to $-32 \mathrm{~km} \mathrm{~s}^{-1}$ (Figure 1). Although HD 10125 is not at the centre of the HI cavity, its eccentric position can easily be explained by considering the stellar proper motion. An arc-like structure is found in all 


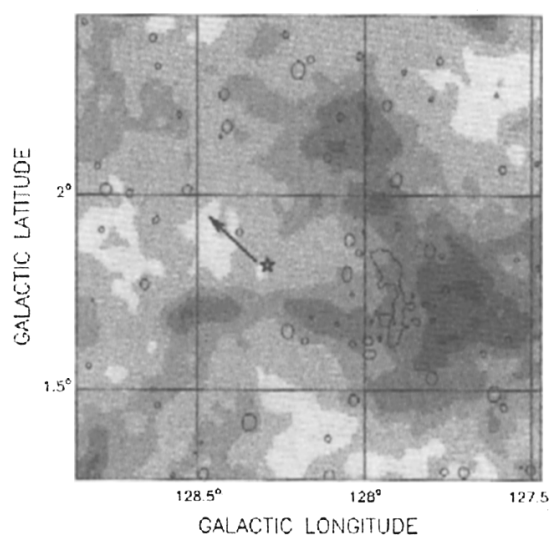

Figure 1. . $6.0 \mathrm{~K}$ radio-continuum contour at $1420 \mathrm{MHz}$ superposed on the average $\mathrm{H}$ I emission (grey-scale). The star symbol indicates the optical position of HD 10125 and the arrow the direction of its proper motion.

the radio continuum data. This structure has an excellent correlation with the observed $\mathrm{H}$ I feature (see Figure 1). The radio continuum emission has a spectral index $\alpha=0.0 \pm 0.1\left(S_{\nu} \propto \nu^{\alpha}\right)$, suggesting a thermal nature for the source.

Based on the 60 and $100 \mu \mathrm{m}$ IRAS-HIRES data a dust temperature image tracing the heated dust is obtained. We found a maximum dust temperature of $33 \mathrm{~K}$ in the area where the continuum emission peaks.

From the $\mathrm{HI}$ data, we derive a kinematical distance of $\sim 3 \mathrm{kpc}$ which is in good agreement with the catalogued distances. This distance was assumed in order to derived the $\mathrm{HI}$, infrared and radio continuum parameteres of the different structures. We conclude that all found features are physically related to each other. The O-type star has enough energetic photons to both ionize the surrounding gas and heat up the dust and, through its powerful wind, also sweep up the $\mathrm{HI}$ and $\mathrm{H}$ II gas.

\section{References}

Münch, G. 1957, ApJ 125, 42

Taylor, A.R. 2001, in: R. Clowes, A. Adamson \& G. Bromage (eds.), The New Era of Wide Field Astronomy, ASP-CS 232, 235

Walborn, N.R. 1971, ApJS 23, 257

Weaver, R.P., McCray, R., Castor, J.I., Shapiro, P.R., Moore, R.L. 1977, ApJ 218, 377 